\title{
Urinary Fistula, CTCAE
}

National Cancer Institute

\section{Source}

National Cancer Institute. Urinary Fistula, CT CAE. NCI Thesaurus. Code C143900.

A disorder characterized by an abnormal communication between any part of the urinary system and another organ or anatomic site. 